\title{
Spatial Pattern and Evolution of Urban System Based on Gravity Model and Whole Network Analysis in the Huaihe River Basin of China
}

\author{
Yong Fan $\left(\mathbb{D},{ }^{1,2}\right.$ Shengdi Zhang, ${ }^{3}$ Zongyi He $\mathbb{D},{ }^{4}$ Biao He ${ }^{D},{ }^{1}$ Haicong Yu, ${ }^{5}$ Xiaoxiao Ye, \\ Hao Yang, ${ }^{4}$ Xiangmin Zhang, ${ }^{2}$ and Zhifeng $\mathrm{Chi}^{2}$ \\ ${ }^{1}$ Research Institute for Smart Cities and Shenzhen Key Laboratory of Spatial Information Smart Sensing and Services, \\ School of Architecture and Urban Planning, Shenzhen University, Shenzhen, China \\ ${ }^{2}$ College of Geographic Sciences, Xinyang Normal University, Xinyang, China \\ ${ }^{3}$ Garmin, Shanghai, China \\ ${ }^{4}$ School of Resource and Environmental Sciences, Wuhan University, Wuhan, China \\ ${ }^{5}$ Center for Assessment and Development of Real Estate, Shenzhen, China
}

Correspondence should be addressed to Biao He; whu_hebiao@hotmail.com

Received 14 January 2018; Revised 9 May 2018; Accepted 28 May 2018; Published 27 June 2018

Academic Editor: Leonid Shaikhet

Copyright (C) 2018 Yong Fan et al. This is an open access article distributed under the Creative Commons Attribution License, which permits unrestricted use, distribution, and reproduction in any medium, provided the original work is properly cited.

\begin{abstract}
The spatial pattern and evolution of urban system have been hot research issues in the field of urban research. In this paper, the network analysis method based on the gravity model and the related measurements were used to reveal the properties of the spatial pattern and evolution of the urban system in the HRB (Huaihe River Basin) of China. The findings of this study are as follows: During the period from 2006 to 2014, the economic contact between the HRB cities has been strengthened, but the differences between cities have been expanding. In general, the HRB cities have not yet formed a close network structure, and a trend of economic integration has not been found. This paper expresses the spatial pattern and evolution of urban system in an intuitive way and helps to explain the evolution mechanism of urban system. The method was confirmed by empirical research. Because of the operational and visual expression, this method has broad application prospects in the urban system research.
\end{abstract}

\section{Introduction}

The spatial pattern and evolution of urban system are important manifestations of the human social development process on a spatial level [1], which reflects the change in the human social spatial structure and reveals the spatial patterns of the overall behavior of human society. These changes and their patterns are the foundations for the research of the sustainable development of human society [2].

Six super-large urban agglomerations have been formed in mainland China: Shanghai-Nanjing-Hangzhou, BeijingTianjin-Tangshan, Pearl River Delta, the central and southern Liaoning Province, Sichuan Basin, and Shandong Peninsula. Six city-and-town-concentrated areas (approximate to urban agglomeration) have also been formed: Central Shanxi Province, Central Hunan Province, Central Plains,
Fuzhou-Xiamen, Harbin-Daqing-Qiqihar, and Wuhan area $[3,4]$. If the geographical positions of urban agglomerations are compared with several main rivers in China (the Changjiang River, the Huanghe River, the Liaohe River, the Huaihe River, the Songhuajiang River, the Pearl River, the Haihe River, and the Minjiang River), it can be found that they mostly have corresponding relations. Only the HRB (Huaihe River Basin) has not formed large scale urban agglomeration [5]. Therefore, this paper wishes to determine the relationship between the HRB cities and puts forward feasible suggestions to promote contact between cities [6].

Population and economic growth are the main driving forces for the growth of urban system [7]. The cellular automata model [8], support vector machine [9], selforganizing structure model [10], gravity model [11], and 
systematic dynamics [12] have been used to describe spatial expansion of cities. The growth of urban systems is also influenced by the scale of urban space, traffic network, and complexity of the terrain [13-15]. A mechanism for the growth of the city has been proposed [16] but modeling the spatial and temporal dynamics of urban formation and evolution remains difficult [17]. Urban systems evolution mainly includes changes in two aspects: land use pattern and system structure [18]. The gravity model has been widely applied in this field. Based on graph theory, large scale analysis has demonstrated that the spatial structure of urban system becomes increasingly effective and stable with increased complexity and more favorable to the sustainable development of the urban system [19].

The formation of urban agglomeration is not only connected with geographical location and distribution of the city but also relevant to the economy and social culture [20]; the cross-border movement of capital, information, products, talented people, and technology is the notable feature of regional economic development $[21,22]$.

From previous literature, we know that network analysis can be used to analyze resource exchange and relation between actors (i.e., individuals, groups, cities, etc.) [23]. Because of this characteristic, network analysis has a great contribution in the field of information exchange [24], group behavior research, and other fields $[25,26]$. However, although network analysis has received great concern in regional economics since the last century [27], only in recent years whole network analysis has been used to study regional economic network structures [28-30]. The method of whole network analysis could be used to study the relationship, especially economic contact, among cities.

Currently, the main focus of whole network urban system is on explicit urban networks (i.e., traffic networks $[14,19]$ ) or the ranking of urban system centers, but there is a lack of dynamic and visual expression on internal membership in urban system [31]. The distinction between an urban system, as a specific geographical cluster, and a city is its complex interactions and connections among network members. Therefore, the internal connection of urban system should be multilateral, interactive, and networked [32].

These studies provide a method foundation for understanding the urban systems evolution. Our goal was to explain the urban system structure based on temporal and spatial evolution and use this to reconstruct the timelines of spatial patterns of regional urban system. Accordingly, in this paper, a modified gravity model is used to construct the network with the location, scale, and economic level data of the HRB cities, and the method of whole network analysis is used to study the urban network structure and the relationships among cities.

\section{Theories and Methods}

2.1. Gravity Model. The gravity model is based on the formula of universal gravitation:

$$
F=\frac{G M_{1} M_{2}}{d^{2}},
$$

which describes the interaction between the two masses $M_{1}$ and $M_{2}$ of objects, where $\mathrm{d}$ is the distance between the two objects and $G$ is the universal gravitation constant.

Similar to the interaction between objects, there is also a relationship between cities in a certain area; in economics, this is called economic gravity theory $[33,34]$.

Because of this similarity, many scholars have attempted to apply the formula of universal gravitation to the measurement of a city's gravity:

$$
F_{i j}=\frac{G M_{i}^{\beta 1} M_{j}^{\beta 2}}{D_{i j}^{\beta 3}},
$$

where $F_{i j}$ denotes the quantity of spatial flows. $M_{i}$ and $M_{j}$ are the GDP (Gross Domestic Product) of a city; $D$ is the distance between two cities; $G, \beta^{1}, \beta^{2}, \beta^{3}$ are constants.

To describe the scale and development degree of a city more scientifically, some scholars have also considered the population and area of a city when constructing the model $[35,36]$.

However, cities are not the same as ordinary objects. The economic impact of city $i$ on city $j$ is not equal to the economic impact of city $j$ on city $i$, and the contributions of the two cities to the economic gravity are also different. Consequently, we defined the constant as the ratio of GDP in city $i$ to the GDP in city $i$ and city $j$, and the modified gravity model is thus obtained:

$$
\begin{aligned}
& F_{i j}=k_{i j} \frac{\sqrt{P_{i} G_{i}} \times \sqrt{P_{j} G_{j}}}{D_{i j}^{2}} \\
& k_{i j}=\frac{G_{i}}{G_{i}+G_{j}},
\end{aligned}
$$

where $P_{i}$ and $P_{j}$ are the registered population in city $i$ and city $j ; G_{i}$ and $G_{j}$ are the GDP in city $i$ and city $j ; D_{i j}^{2}$ is the distance between city $i$ and city $j ; k_{i j}$ is the contribution rate from city $i$ to city $j$. If the city is at a prefecture level or above, the statistical data do not include the counties under the jurisdiction of that city.

First, We calculated the $F_{i j}$ of all the cities in the HRB based on (3) and showed the result as a matrix. Then, urban system network structure of the HRB was calculated and analyzed using Ucinet Platform.

\subsection{Whole Network Analysis}

2.2.1. Density. Network Density refers to the closeness degree among the network members: the closer the members, the higher the density of the network. For a binary network, its density is the ratio of the number of actual relationships in the network to the theoretical maximum number of relationships, whereas for a multivalued network, such as the network analyzed in this paper, its density is the average of all contact values except self-contact:

$$
D=\sum_{i} \sum_{j, i \neq j} \frac{F_{i j}}{\left(n^{2}-2 n\right)},
$$


where $F_{i j}$ is the economic impact of city $i$ on city $j$; $n$ is the number of cities in the urban network.

\subsubsection{Degree Centrality and Centralization Measurement.} Degree centrality analysis is used to explore how central the members are in the network: the higher the degree centrality, the more important the member in the network [37]. Generally, degree centrality includes the absolute value and the standard value, the latter of which is used to compare between different network; thus, we use the absolute value of degree centrality in this analysis $[38,39]$.

Because what we construct is a directed network, each node has an out-degree and an in-degree, which represent the extent of a city's influence on other cities and the extent to which a city is affected by other cities, respectively:

$$
C_{A D i}= \begin{cases}\sum_{j} F_{i j} & \text { (outdegree) } \\ \sum_{j} F_{j i} & \text { (indegree }),\end{cases}
$$

where $C_{A D i}$ is the degree centrality of city $i$ and $F_{i j}$ is the economic impact of city $i$ on city $j$.
Centralization is used to describe the overall centrality of a network; namely, it compares the highest degree of centrality in the network with the other members' centrality:

$$
C_{A D}=\frac{\sum_{i}\left(C_{\max }-C_{A D i}\right)}{C_{\max }},
$$

where $C_{\max }$ is the highest degree of centrality of all cities in the agglomeration and $C_{A D i}$ is the degree centrality of city $i[40]$.

2.2.3. Measurement of Subgroups. A subgroup refers to a number of closely related members in a network. This analysis is usually used to study the number of subgroups in the network, the relation of the internal members of a subgroup, and the relation among the subgroups. It is not conducive to the network development if there are many distantly related subgroups [41].

In this paper, we used the CONvergence of iterated CORrelations (CONCOR) algorithm to analyze the network:

$$
r_{i j}=\frac{\sum\left(x_{k i}-\overline{x_{\bigcirc i}}\right)\left(x_{k j}-\overline{x_{\bigcirc j}}\right)+\sum\left(x_{i k}-\overline{x_{i \circ}}\right)\left(x_{j k}-\overline{x_{k \circ}}\right)}{\sqrt{\sum\left(x_{k i}+\overline{x_{\bigcirc i}}\right)^{2}+\sum\left(x_{i k}+\overline{x_{i \circ}}\right)^{2}} \sqrt{\sum\left(x_{k j}+\overline{x_{\odot j}}\right)^{2}+\sum\left(x_{j k}+\overline{x_{j \circ}}\right)^{2}}},
$$

where $\overline{x_{i \circ}}\left(\overline{x_{j 0}}\right)$ is the value in row $i(j)$ of the matrix and $\overline{x_{\odot i}}\left(\overline{x_{\odot j}}\right)$ is the value in column $i(j)$ of the matrix. At the initial level of analysis, CONCOR calculates using the above equation until all values converge to either 1 or -1 , resulting in all nodes being grouped into one of two categories [33].

2.2.4. Structural Equivalence Measurement. Structural equivalence means that if two members in a network swap their positions, the network structure will not change:

$$
\begin{aligned}
& F_{i k}=F_{j k}, \\
& F_{k i}=F_{k j} .
\end{aligned}
$$

Certainly, it is very difficult to find fully structural equivalence between two members in a network. This measurement can be used to develop a general analysis of the network structure to better understand the similarities among the members.

\section{Data Source and Processing}

3.1. Study Area. Historically, the HRB has experienced several changes, and this has led to a variety of versions of HRB cities. In this paper, to make the study area more scientific and standard, we use the "HRB and Shandong Peninsula map" provided by the Huaihe River Water Resource Network, and the research area is defined according to the basin boundary.
According to the latest administrative plan in 2016, the HRB covers 1 provincial city, 27 prefecture-level cities, and 139 county-level cities (Figure 1).

3.2. Data Source. In this paper, the collected data include the registered population and GDP of the cities in the study area in the years of 2006, 2010, and 2014, and data of the cities at the prefecture level or above do not include the counties under the jurisdiction of that city.

The main sources of data are statistical yearbook of provinces, the national economic and social development statistical bulletin of counties, and the government work report. Because we use the latest administrative plan in 2016, if there were previously county-level cities that then became a district of a prefecture-level city, the data on the county-level city are counted as a part of the prefecture-level city.

3.3. Missing Data and Processing Method. Missing data include the GDP of Juxian and Feixian (both in Shandong Province) in 2006 and registered population of all cities at or above the county level in Henan Province in 2010:

GDP of Juxian in 2006: using GDP in 2005 and annual average growth rate from 2005 to 2010 (from the government five-year plan report) to simulate the data.

GDP of Feixian in 2006: using GDP in 2005 and annual growth rate from 2005 to 2006 of Yinanxian (both counties under the jurisdiction of Linyi, and they had similar GDP in 2005) to simulate the data. 


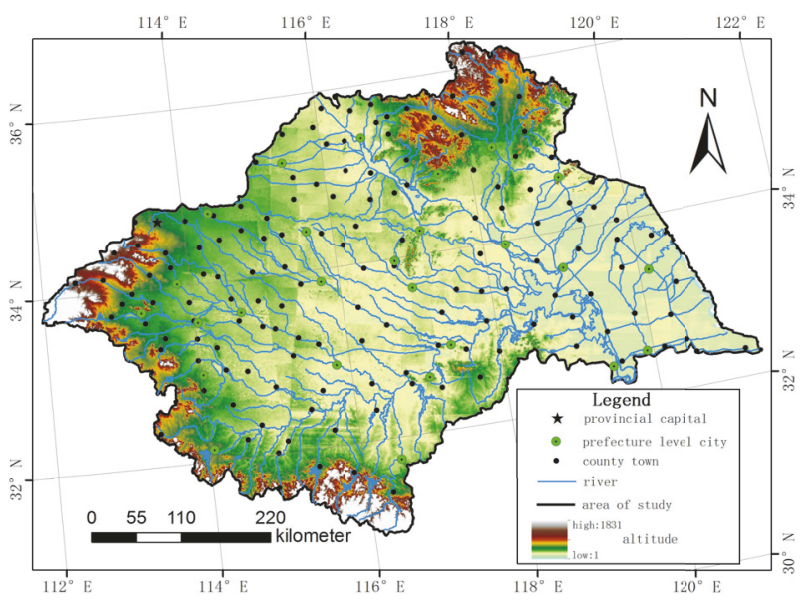

FIGURE 1: Study area.

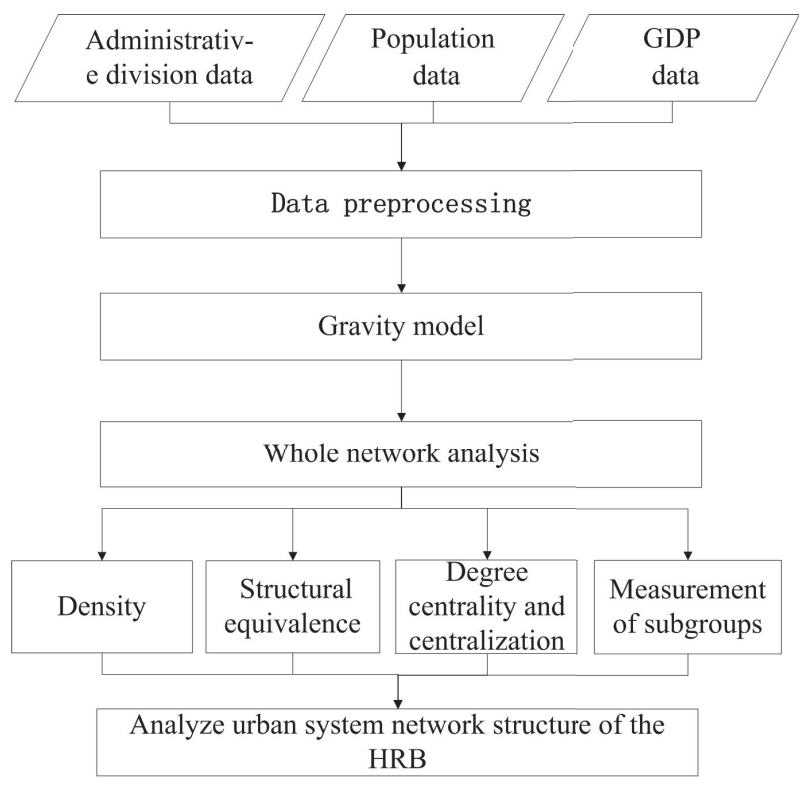

FIGURE 2: The process of whole network analysis.

Registered population of Henan Province cities in 2010: taking the average registered population in 2009 and 2011.

3.4. The Process of Whole Network Analysis. Figure 2 gave the process of the whole network analysis. First, We collected population, GDP, and administrative division data of the cities in the study area. Then, we used ArcGIS 10.0 software to calculate the distance between cities. Third, we introduced data of the registered population, GDP, and distance used in formula (3) to calculate the value of economic contact between cities and then convert the result into the form of a matrix. At last, urban system network structure of the HRB was calculated and analyzed using Ucinet Platform.

\section{Whole Network Analysis of the HRB Cities}

4.1. Whole Network Density. Table 1 shows the details about the density analysis result of the HRB urban network. We can
TABLE 1: Density of the HRB urban network.

\begin{tabular}{lccc}
\hline Year & $\mathbf{2 0 0 6}$ & $\mathbf{2 0 1 0}$ & $\mathbf{2 0 1 4}$ \\
\hline Avg Value & 0.3243 & 0.6466 & 1.0891 \\
Std Dev & 2.1423 & 4.3893 & 7.2752 \\
\hline
\end{tabular}

TABLE 2: Statistical table of critical values of the HRB cities.

\begin{tabular}{lccccc}
\hline $\begin{array}{l}\text { Year } \begin{array}{l}\text { critical } \\
\text { value }\end{array} \\
2006\end{array}$ & $\mathbf{1}$ & $\mathbf{2}$ & $\mathbf{3}$ & $\mathbf{4}$ & $\mathbf{5}$ \\
2010 & 4147 & 2312 & 1576 & 1183 & 952 \\
2014 & 4128 & 2283 & 1557 & 1171 & 946 \\
\hline Ratio & 4160 & 2327 & 1574 & 1180 & 957 \\
\hline
\end{tabular}

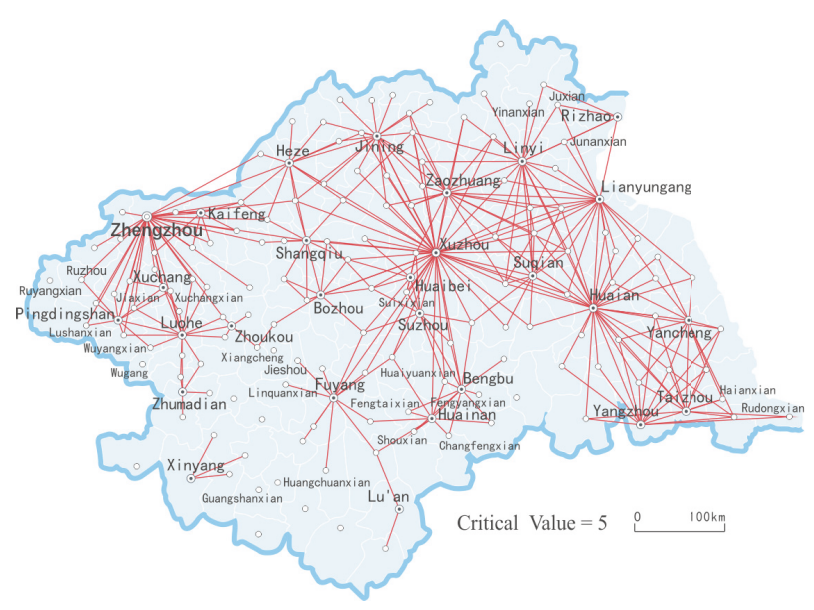

FIGURE 3: Urban network structure in 2014 (at the prefecture level or above).

find that the density of the urban network grew rapidly; this is mainly because in this period, with the rapid development of the economy, the economic contact between the cities of the HRB greatly increased. Meanwhile, the growth rate of the standard deviation was far greater than that of the average value, which shows that the economic difference between cities was increasing, and the increase in economic contact value was mainly in the large cities. The unbalanced development of the city economy in the whole HRB had an intensifying trend.

If the density of each year has critical value equal to 1 , double the density has critical value equal to 2 , etc. The statistical results of the number of cities above the critical value are shown in Table 2 and the urban network structure when the critical value is 5 in 2014 is shown in Figures 3 and 4. It can be seen that the county-level cities were likely to have strong economic contact only when they were fairly close, and high economic impacts produced by cities at the prefecture level or above raised the whole network density.

Figures 3 and 4 show the general structure of urban economic linkage intensity in HRB. Overall, only in the 
TABLE 3: Statistical table of Huaihe Basin's top three out-degree cities.

\begin{tabular}{lcccccccc}
\hline \multirow{2}{*}{ Name } & \multirow{2}{*}{ Province } & \multicolumn{2}{c}{ Prefecture- level City } & \multicolumn{3}{c}{ In-degree } & \multicolumn{3}{c}{ Out-degree } \\
& & & $\mathbf{2 0 0 6}$ & $\mathbf{2 0 1 0}$ & $\mathbf{2 0 1 4}$ & $\mathbf{2 0 0 6}$ & $\mathbf{2 0 1 0}$ & $\mathbf{2 0 1 4}$ \\
\hline Xuzhou & Jiangsu & $/$ & 68.3 & 135.5 & 242.7 & 436.1 & 839.3 & 1373.6 \\
Zhengzhou & Henan & $/$ & 57.4 & 114.9 & 211.6 & 340.9 & 749.9 & 1308.6 \\
Huaibei & Anhui & $/$ & 130.6 & 279.9 & 480.5 & 279.0 & 589.1 & 945.5 \\
\hline
\end{tabular}

TABLE 4: Statistical table of several cities of the HRB.

\begin{tabular}{|c|c|c|c|c|c|c|c|c|}
\hline \multirow{2}{*}{ Name } & \multirow{2}{*}{ Province } & \multirow{2}{*}{ Prefecture- level City } & \multicolumn{3}{|c|}{ In-degree } & \multicolumn{3}{|c|}{ Out-degree } \\
\hline & & & 2006 & 2010 & 2014 & 2006 & 2010 & 2014 \\
\hline Xuchangxian & Henan & Xuchang & 178.6 & 402.1 & 300.3 & 197.8 & 324.6 & 695.8 \\
\hline Xuchang & Henan & 1 & 187.7 & 322.9 & 238.2 & 145.1 & 353.4 & 445.8 \\
\hline Xiangcheng & Henan & Zhoukou & 63.1 & 122.2 & 227.8 & 92.4 & 157.4 & 225.8 \\
\hline Zhoukou & Henan & I & 54.6 & 106.9 & 179.2 & 47.1 & 85.1 & 161.7 \\
\hline
\end{tabular}

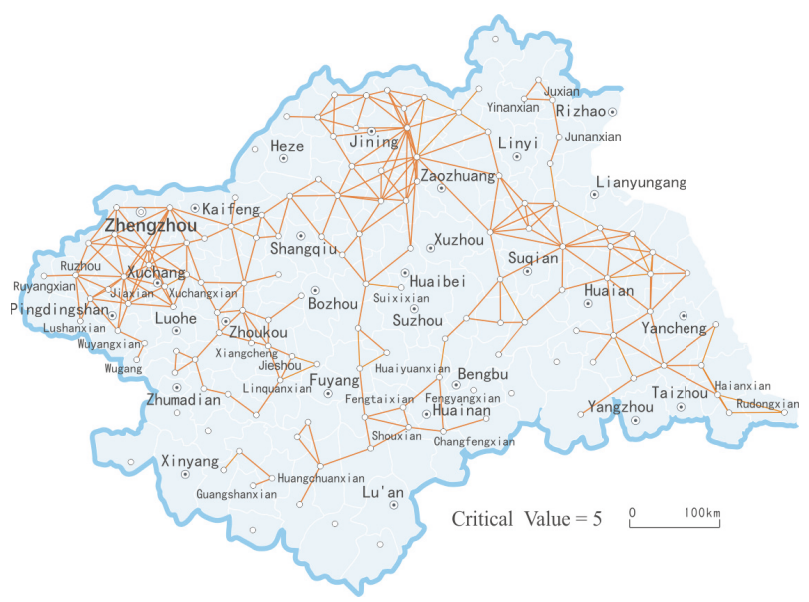

FiguRE 4: Urban network structure in 2014 (at the county level).

HRB urban network, compared with other parts, the economic contact intensity of southwest cities was weak; even prefecture-level cities could not easily produce strong economic influence.

\subsection{Degree Centrality and Centralization Measurement.} Details of the analysis result of degree centrality can be seen in Figure 5 which shows the degree centrality analysis result of the HRB cities in 2014. Through calculation, the average in-degree (out-degree) is 180.8; hence, we used the average, 1.5 times the average, and 2 times the average to partition the data classification.

The analysis results show the following:

(1) Cities with high out-degree values were mainly concentrated near the province junction (Jining-ZaozhuangXuzhou-Huaibei) and part of Henan Province (ZhengzhouLuohe-Xuchang), whereas the southwest and south of the HRB were small in number. The distribution of the cities with high in-degree value was similar to that of high out-degree cities but larger in number. From the perspective of color depth distribution, we can find that cities' in-degree values were closer to the average.

(2) The highest out-degree cities were Xuzhou, Zhengzhou, and Huaibei, which shows that these three were relatively economically developed cities in the HRB. Among these three cities, the in-degree and out-degree centrality values of Xuzhou and Zhengzhou were similar: the out-degree values were very high, whereas the in-degree values were only approximately $1 / 6$ of the out-degree. This shows that although these two cities had great economic power, their economic radiations with the surrounding cities were limited, and they did not establish economic contact actively. In contrast to the above two cities, the values of out-degree and in-degree of Huaibei were relatively close, which reflects the fact that Huaibei had stronger economic contact with neighboring cities (Table 3 ).

(3) Because of the difference in the economic aggregate, generally, a prefecture-level city's out-degree is greater that its in-degree, whereas the county-level cities are the opposite. There were special circumstances in 2006. The main reason for this phenomenon was that the two prefecturelevel cities' urban areas were smaller with lower population, resulting in reduced economic influence compared with the surrounding counties. However, there was a difference in the two prefecture-level cities' development after 2006: the out-degree of Xuchang rose after 2006, and Xuchangxian's out-degree became less than its in-degree, which shows that Xuchang transferred some core industries to the city center and enhanced its economic influence. However, Zhoukou maintained its original development policy, and there were few changes in the contrast of out-degree and in-degree (Table 4).

Through the comparison of the out-degree centralization and in-degree centralization of the HRB urban network, it can be found that both out-degree and in-degree centralization are small, which indicates that the whole centrality of the urban network was low (the difference in out-degree and in-degree between cities is little), and the network structure was loose. This also shows that there were few core cities 


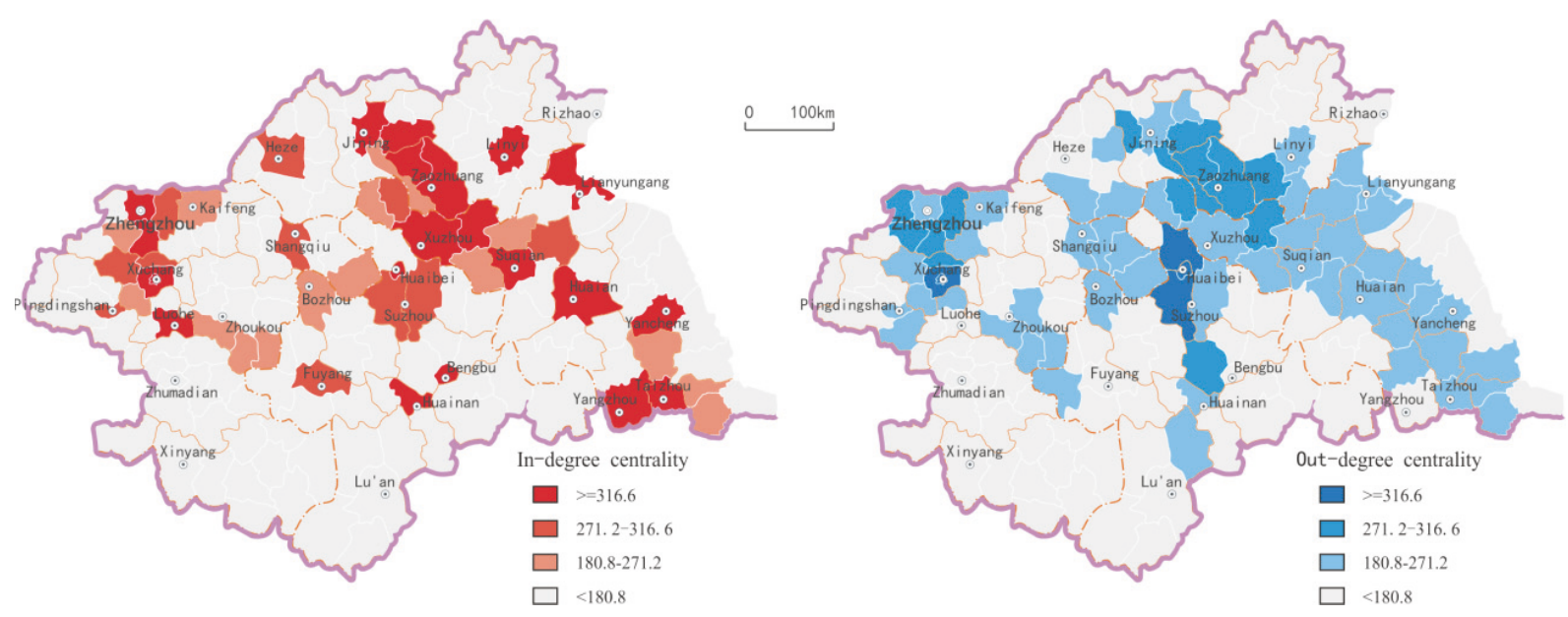

FIGURE 5: Degree centrality analysis results of the HRB cities in 2014.

TABLE 5: Statistical table of the urban network centralization of the HRB.

\begin{tabular}{ccc}
\hline Year & Out-degree Centralization & In-degree Centralization \\
\hline 2006 & $1.169 \%$ & $0.615 \%$ \\
2010 & $1.050 \%$ & $0.618 \%$ \\
2014 & $1.062 \%$ & $0.621 \%$ \\
\hline
\end{tabular}

that could promote the whole urban network's economic development in the HRB.

The out-degree centralization from 2006 to 2010 was in decline. The reason may be that some large cities drove the development of the small cities around in the economy, thus bridging the gap of out-degree between cities. Meanwhile, the in-degree centralization slightly increased, which illustrates that the economic development appeared only in some cities, and the urban network lacked core cities such that the economic radiation range of the developed cities was low. From 2010 to 2014, both the out-degree and the in-degree centralization rose slightly. The potential reason may be that central cities strengthened ties with each other, causing economic mutual promotion; at the same time, some small cities introduced external resources actively to develop the economy and opened the gap with other small cities (Table 5).

4.3. Measurement of Subgroups. Through the subgroup analysis with the CONCOR algorithm, we obtained the result of 8 subgroups (based on 3-plex). Figure 6 shows the analysis result of the subgroups in 2014.

It is shown in Figure 6 that, in 2014, the subgroups of the HRB include Subgroup 1 (center: Zhoukou-ZhumadianXinyang), Subgroup 2 (center: Bozhou- Fuyang-Lu'an), Subgroup 3 (center: Zhengzhou-Pingdingshan-Xuchang Luohe), Subgroup 4 (center: Kaifeng-Shangqiu-Heze), Subgroup 5 (center: Jining-Xuzhou-Huaibei- Suzhou), Subgroup 6 (center: Rizhao-Linyi-Zaozhuang), Subgroup 7 (center: Taizhou- Yangzhou-Huaian-Lianyungang), and Subgroup 8 (center: Suqian-Bengbu -Huainan).

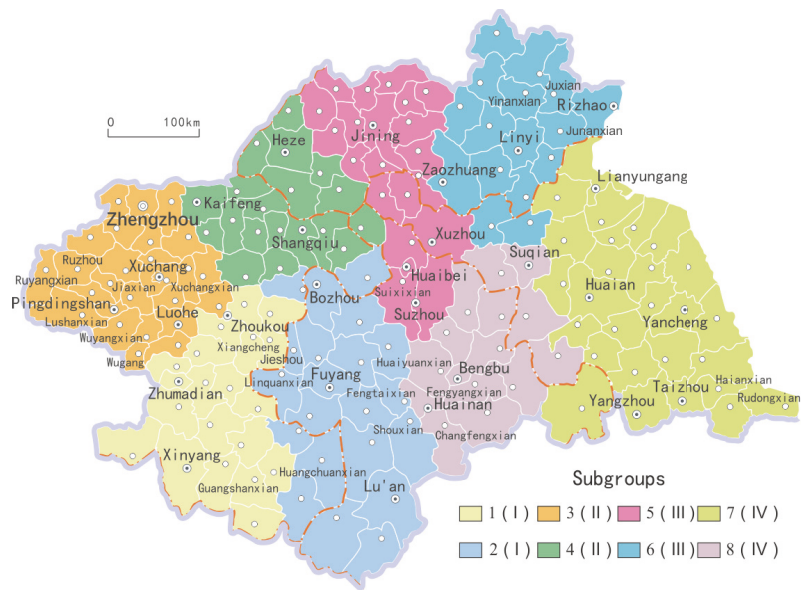

FIgURE 6: The distribution of subgroups of the HRB in 2014.

(In Figure 6, Roman numbers in brackets I, II, III, and IV represent the analysis result based on 2-plex, and Arabic numerals 1 to 8 represent the analysis result based on 3-plex; we mainly use the latter.)

During the study period, there were few changes of the subgroup structures. The subgroup structure in 2010 and that in 2014 were exactly the same, and in 2006, there was only one difference from the other two years; Shouxian (under the jurisdiction of Lu'an in Anhui Province) belonged to Subgroup 8, but in 2010 and 2014, it belonged to Subgroup 2. This shows that the industrial structure of the HRB did not change significantly.

The formation of subgroups was mainly related to the distance, not only membership in the same province. Most subgroups in the urban network crossed 2 or 3 provinces, and this shows low relevance of the cities in the same province.

From 2006 to 2014, the density of all subgroups had an upward trend (see Tables 6, 7, and 8 for details). In the three tables, the numbers in bold and underlined indicate that the subgroup density is greater than the whole network density, 
TABLE 6: Subgroup density table of the HRB urban network in 2006.

\begin{tabular}{lcccccccc}
\hline $\mathbf{2 0 0 6}$ & $\mathbf{1}$ & $\mathbf{2}$ & $\mathbf{3}$ & $\mathbf{4}$ & $\mathbf{5}$ & $\mathbf{6}$ & $\mathbf{7}$ \\
\hline 1 & $\underline{\mathbf{0 . 8 9}}$ & 0.23 & 0.18 & 0.12 & 0.04 & 0.02 & 0.01 & 0.04 \\
2 & 0.24 & $\underline{\mathbf{0 . 7 8}}$ & 0.06 & 0.21 & 0.15 & 0.05 & 0.03 & 0.24 \\
3 & 0.30 & 0.11 & $\underline{\mathbf{2 . 1 0}}$ & 0.32 & 0.07 & 0.04 & 0.02 \\
4 & 0.14 & 0.17 & 0.20 & $\underline{\mathbf{1 . 0 7}}$ & 0.24 & 0.06 & 0.02 & 0.04 \\
5 & 0.09 & 0.27 & 0.08 & $\underline{\mathbf{0 . 4 4}}$ & $\underline{\mathbf{2 . 8 8}}$ & $\underline{\mathbf{0 . 6 2}}$ & 0.13 & \\
6 & 0.04 & 0.10 & 0.04 & 0.13 & $\underline{\mathbf{0 . 5 5}}$ & $\underline{\mathbf{1 . 8 9}}$ & 0.29 \\
7 & 0.03 & 0.08 & 0.03 & 0.05 & 0.10 & 0.29 & $\underline{\mathbf{1 . 4 9}}$ \\
8 & 0.05 & 0.29 & 0.03 & 0.06 & 0.13 & 0.16 & 0.25 \\
\hline
\end{tabular}

TABLE 7: Subgroup density table of the HRB urban network in 2010.

\begin{tabular}{lcccccccc}
\hline $\mathbf{2 0 1 0}$ & $\mathbf{1}$ & $\mathbf{2}$ & $\mathbf{3}$ & $\mathbf{4}$ & $\mathbf{5}$ & $\mathbf{6}$ & $\mathbf{7}$ \\
\hline 1 & $\underline{\mathbf{1 . 6 9}}$ & 0.44 & 0.34 & 0.24 & 0.07 & 0.03 & 0.02 & 0.07 \\
2 & 0.45 & $\underline{\mathbf{1 . 6 2}}$ & 0.12 & 0.38 & 0.28 & 0.09 & 0.07 & 0.04 \\
3 & 0.57 & 0.21 & $\underline{\mathbf{4 . 1 9}}$ & $\underline{\mathbf{0 . 6 6}}$ & 0.14 & 0.49 & 0.12 & 0.05 \\
4 & 0.26 & 0.33 & 0.40 & $\underline{\mathbf{2 . 0 9}}$ & $\underline{\mathbf{0 . 8 6}}$ & $\underline{\mathbf{5 . 7 0}}$ & $\underline{\mathbf{1 . 1 8}}$ & 0.10 \\
5 & 0.17 & 0.53 & 0.15 & 0.24 & $\underline{\mathbf{0 . 9 6}}$ & $\underline{\mathbf{3 . 4 7}}$ & 0.26 \\
6 & 0.08 & 0.18 & 0.07 & 0.11 & 0.22 & 0.64 & $\underline{\mathbf{3 . 1 8}}$ \\
7 & 0.07 & 0.16 & 0.05 & 0.13 & 0.28 & 0.39 & 0.39 \\
8 & 0.10 & $\underline{\mathbf{0 . 6 8}}$ & 0.05 & & & 0.63 \\
\hline
\end{tabular}

TABLE 8: Subgroup density table of the HRB urban network in 2014.

\begin{tabular}{|c|c|c|c|c|c|c|c|c|}
\hline 2014 & 1 & 2 & 3 & 4 & 5 & 6 & 7 & 8 \\
\hline 1 & $\underline{2.85}$ & 0.72 & 0.57 & 0.38 & 0.11 & 0.05 & 0.04 & 0.11 \\
\hline 2 & 0.74 & $\underline{2.64}$ & 0.20 & 0.64 & 0.46 & 0.14 & 0.11 & 0.62 \\
\hline 3 & 0.92 & 0.34 & $\underline{6.90}$ & $\underline{1.17}$ & 0.24 & 0.12 & 0.07 & 0.13 \\
\hline 4 & 0.44 & 0.55 & 0.68 & $\underline{3.58}$ & 0.86 & 0.20 & 0.08 & 0.17 \\
\hline 5 & 0.28 & 0.89 & 0.25 & $\underline{1.48}$ & $\underline{9.43}$ & $\underline{1.92}$ & 0.43 & 1.05 \\
\hline 6 & 0.14 & 0.30 & 0.12 & 0.39 & 1.59 & $\underline{5.49}$ & 0.98 & 0.97 \\
\hline 7 & 0.12 & 0.28 & 0.09 & 0.19 & 0.39 & $\underline{1.11}$ & $\underline{5.54}$ & $\underline{1.13}$ \\
\hline 8 & 0.17 & 1.08 & 0.09 & 0.23 & 0.52 & 0.75 & 0.74 & $\underline{4.94}$ \\
\hline
\end{tabular}

indicating that the links between the two subgroups are relatively close. It can be seen that, during the study period, the links within each subgroup were closely related, but links between subgroups were loose. In 2006, only three densities of the links between subgroups are greater than the network density. This situation improved after 2006: the number of the closely related subgroups grew gradually, showing that subgroups were establishing economic contact initiatively.

As shown in Table 6, the strongest link within a subgroup in 2006 is in Subgroup 5, and according to the data of 2010 and 2014, we can find that this strong economic tie continued. The analysis result shows that Subgroup $5 \mathrm{did}$ not only have close internal ties but had a strong economic impact on Subgroup 4; meanwhile, it had mutual economic influence on Subgroup 6.

In 2006, the subgroups with the weakest internal links were Subgroup 1 and Subgroup 2 which are located in the southwest of the HRB. Table 6 shows that despite the two subgroups' weak self-contact, they developed in a very isolated way in the urban network, and this situation was not improved until 2014.

Although subgroup 3 was in close contact with itself and had good economic development in 2006, it did not have any significant economic relationship with any other subgroups. However, we can see from Table 7 that, in 2010, Subgroup 3 had an obvious economic influence on Subgroup 4. This might be because, from 2006 to 2010, Zhengzhou, the provincial capital of Henan Province, developed rapidly and enhanced its economic radiation capacity, resulting in more close ties with other cities in Henan Province. During this period, contact between Subgroup 2 and Subgroup 8 also enhanced, but such economic contact weakened in 2014; the reason for this may be that there were few high-out-degreevalue cities in Subgroup 2 and Subgroup 8, rendering the two subgroups unable to establish frequent economic exchanges due to the lack of central cities.

From 2010 to 2014, Subgroup 7's economic influence improved and had an important impact on the adjacent two subgroups (Subgroup 6 and Subgroup 8). 
TABLE 9: Structural analysis results of the HRB cities.

\begin{tabular}{|c|c|c|c|c|}
\hline & City 1 & Prefecture-level city & City 2 & Prefecture-level city \\
\hline \multirow{13}{*}{$\begin{array}{l}\text { Over the years } \\
\text { Structural } \\
\text { equivalence } \\
>90 \%\end{array}$} & Yishuixian & Linyi & Juxian & Rizhao \\
\hline & Yinanxian & Linyi & & \\
\hline & Haianxian & Nantong & Rudongxian & Nantong \\
\hline & Suixixian & Huaibei & Huaibei & 1 \\
\hline & Guangshanxian & Xinyang & Huangchuanxian & Xinyang \\
\hline & Jieshou & Fuyang & Linquanxian & Fuyang \\
\hline & Huaiyuanxian & Bengbu & Fengyangxian & Tuzhou \\
\hline & Fengtaixian & Huainan & Shouxian & Huainan \\
\hline & Changfengxian & Hefei & Huainan & 1 \\
\hline & Wuyangxian & Luohe & Wugang & Pingdingshan \\
\hline & Lushanxian & Pingdingshan & Jiaxian & Pingdingshan \\
\hline & Ruhou & Pingdingshan & Ruyangxian & Luoyang \\
\hline & Xuchang & 1 & Xuchangxian & Xuchang \\
\hline Added in 2014 & Shangshuixian & Zhoukou & Zhoukou & 1 \\
\hline
\end{tabular}

Overall, until 2014, in addition to Subgroup 1 and Subgroup 2, the other subgroups essentially established a link with one or two subgroups. However, except for the strong mutual influence between Subgroup 6 and Subgroup 5 , most links were one-way, which was not conducive to the development of the entire urban network.

To better integrate Subgroup 1 and Subgroup 2 into the urban network of the HRB, the economic contact between the two subgroups should be strengthened; meanwhile, there should be other subgroups with economic impact on the two subgroups. It can be found in Table 8 that both Subgroup 3 to Subgroup 1 and Subgroup 8 to Subgroup 2 had strong development potential.

To promote the economic integration of the HRB, each subgroup should first positively develop its own economic integration and strengthen its internal economic contact; on this basis, economic exchanges and economic cooperation should be strengthened among subgroups.

4.4. Structural Equivalence Measurement. In this paper, we used correlation to analyze the structural equivalence of the HRB cities. We chose $90 \%$ as the selection criterion, and the analysis results can be seen in Table 9. We can find that there were few differences in the cities with structural similarities from 2006 to 2014, with only the addition of one group in 2014.

Urban spatial structure, a type of economic contact, along with the industrial structure, influences the economic development of the urban network; thus, the economic structure can reflect the situation of industrial structure to some extent. Therefore, the higher the similarity of the urban spatial structure, the greater the possibility of their industrial structure convergence. However, for a region's economic development, it is not suitable to have many cities with similar industrial structures because this is not conducive to product differentiation and complementarity, thus affecting the strength of the city's economic exchanges and cooperation (there are more frequent exchanges between cities with complementary products). This is called structural waste of the same type and is disadvantageous to regional economic integration.

The structural equivalence cities shown in Table 9 can be divided into three cases: county and its prefecture-level city, both counties belonging to the same prefecture-level cities, and adjacent cities. The reason for the former two may be that a prefecture-level city transferred part of its industry to the county, and the development direction of the county is generally determined by the prefecture-level city to which it belongs. The reason for the last one may be that the adjacent counties all had resource-based economies (i.e., mineral resources) or were placed in the same direction after mutual learning.

Structural equivalence cities were mainly concentrated in the Henan and Anhui Provinces, reflecting the urban planning problems of the two provinces. This may also be one of the main reasons for the weak internal relations of Subgroup 1 and Subgroup 2 (the other reason is that there were no cities with great economic influence in the two subgroups).

For the economic development of the whole HRB, the level of structural equivalence of the HRB cities should be reduced to strengthen the economic cooperation and communication between the cities.

\section{Discussion}

5.1. Urban System Network Analysis. With passage of time and city expansion, the exchange of material, energy, and information among cities is inevitable. And this exchange promoted the evolution of urban system. As the urban system is a self-organized system, changes in spatial patterns will normally be regular and gradual. We believe that this change is similar to network change and developed the urban system network analysis method for urban system evolution.

We modified gravity model, which is used to construct the urban system network, and established a system for the study 
of urban system spatial pattern. We then proposed the whole network analysis method to analyze the network structure of urban system and the relationship among cities. Finally, the data of the HRB cities location, scale, and economic level were used to explore the process of urban system evolution. The urban spatial pattern can be expressed by the urban system network, and the urban system development is dependent on the growth of urban scale. The urban system network can help explain the pattern and evolution of urban system. Urban scale growth is the driving force for the urban system development, and its spatial development depends on the links between the cities, that is, the pathways for energy, material, and information exchange. In the development process of regional cities, the connection between cities is determined by the gravitation factor. By evaluating all the city connections within a given region, an overall spatial connection can be established. Urban system network which represents the urban spatial structure can be established. Comparing the urban system network of various times allows the evolution of the urban system spatial pattern to be explored. The urban system network is a new perspective to help illustrate the urban system spatial patterns and the evolution mechanisms.

We have successfully constructed the urban system network for the urban system in the Huai River Basin using the relevant data in 2006, 2010, and 2014. We were able to explain the spatial pattern and probable evolution mechanism of the HRB urban system. This study also provided an answer for the three questions mentioned above concerning the urban system spatial structure and the evolution mechanism of the spatial pattern.

The urban system network expresses the material, energy, and information exchange among cities in a simple visual manner, making it possible that the urban system structure and the spatial relationship between cities are understood intuitively. On this basis we can conduct urban fractal analysis, city statistical analysis, and research on the urban development mechanism and state evolution.

5.2. The Cities of the HRB. According to all analysis results, we can find that the urban network structure of the HRB was not close and the economic integration level was not high. During the study period, the whole network density increased, indicating the gradual growing of the economic links between cities; however, the increase in the standard deviation of the whole network density also shows that the development differences of the HRB network were expanding. Degree centrality analysis determined the cities with high out-degree values (i.e., Xuzhou and Zhengzhou); their in-degree values were far less than their out-degree values, indicating that they did not promote the integrated development of the surrounding cities. Both in-degree and out-degree centralization were low from 2006 to 2014, showing that there were few core cities in the HRB. There were 8 subgroups in the HRB, each of which had a tight internal economic tie, but the relationships among the subgroups still needed to be strengthened.

Overall, there was no obvious trend of economic integration in the HRB. Some subgroups were still isolated in the urban system network, and although the other subgroups were actively establishing connections, these connections were mostly one-way and could not spread across the network. There were a number of cities with structural equivalence, and the number was growing; high industrial overlap existed between cities, and there was no formation of industrial differences and complementarity. There were no central cities in the southwest of HRB; the economic influence of the relatively developed cities could not reach the southwest. Therefore, we have reached a similar conclusion: the central city of the HRB is still in the primary stage of evolution and the urban system has not yet formed a close network structure.

Based on the above analysis, in order to form a close urban system of the HRB more quickly and achieve economic integration at an early date, the following points are put forward:

First, the establishment of core cities can promote the overall development of the HRB. Xuzhou, which is located in the center of the HRB and had highest out-degree value, could be chosen to be the core city; there were also several cities in the city circle. Measures can be taken such as reducing the trans-provincial and trans-city transaction costs to actively promote the development of the surrounding cities.

Second, establish a regional core city in the southwestern part of HRB. This can strengthen the cities economic exchanges in the southwest of HRB and allow the southwest to claim this city as the center to establish economic cooperation with other parts of the HRB; this could also prevent the southwest region from being isolated from the rest of the HRB development.

Third, expand the economic influence of the cities near the border of the southwest in the HRB. From subgroup analysis, we could find that the greatest impacts on the southwest region (Subgroup 1 and Subgroup 2) were Subgroup 3 and Subgroup 8. To enhance this influence, methods can be implemented such as strengthening the economic influence of Luohe and Huainan so that the connection between subgroups will grow and thus increase the strength of the urban network in the HRB. The economic impact of Huaibei could also be strengthened, letting Huaibei become the economic radiation window for northeast to southwest HRB.

Fourth, we should also pay attention to the differential development when focusing on the economy. A qualified core city should not only play an active role in economic development but also drive the differential industrial development of surrounding cities to avoid vicious competition. At this point, cities could develop new industries, such as the service industry or tourism.

5.3. Uncertainty Analysis and Improvement. Alternatively, we can directly use the night light data [42] to describe the urban scale; the night light data can reflect the spatial geographic information and intensity change procession, which can reflect the urban area, population, GDP, and other perspectives of the city scale [43]. We can also use the average time between two cities (taking the train or the highway) instead of considering the two cities by the straight line distance analysis. However, because our analysis was based on the county-level cities, there were limitations on the data. 
If we have enough data, we could also add geodesic distance analysis and the measurement of betweenness centrality; the former studies the traffic development level and the urban network connection strength, and the latter can research the ability of a central city to control other cities using a particular form of diagrammatic sociospatial knowledge [39].

\section{Conclusions}

The urban system network and the model of gravitation to urban system spatial growth were proposed in this study, and urban system in the HRB was constructed with the method to analyze the evolution mechanism of urban system. We come to the following conclusions:

(1) The spatial structure of urban system can be expressed by the network. The urban system network spatial extension direction is determined by the gravitation of urban system spatial growth, and the spatial growth process of urban system network reveals the evolution mechanism of urban system.

(2) Urban system in the HRB has experienced slow development with uneven temporal and spatial distribution. During the period from 2006 to 2014, the economic contact between cities in the HRB was strengthened, but the differences in intercity development were expanding; cites in the HRB have not yet formed a close network structure, and the trend of economic integration has not been found. We propose to replan the sustainable development of urban system in the HRB.

(3) The urban system network explains the evolution mechanism of the urban system, and the empirical research confirms this method. Because of its operability and visual expression, this method has broad application prospect for further study of urban system.

\section{Data Availability}

All data generated or analyzed during this study were included in this manuscript.

\section{Conflicts of Interest}

The authors declare that there are no conflicts of interest regarding the publication of this paper.

\section{Acknowledgments}

Minmin Li gave many suggestions in English expression and writing. This work was supported by the Natural Science Foundation of China (Grant no. 41701187) and project funded by China Postdoctoral Science Foundation (Grant no. 197675).

\section{References}

[1] B. Catalán, D. Saurí, and P. Serra, "Urban sprawl in the Mediterranean? Patterns of growth and change in the Barcelona Metropolitan Region 1993-2000," Landscape and Urban Planning, vol. 85, no. 3-4, pp. 174-184, 2008.
[2] J. A. Alnsour, "Managing urban growth in the city of Amman, Jordan," Cities, vol. 50, pp. 93-99, 2016.

[3] S. M. Yao, X. H. Wang, and Z. G. Chen, "Strategy problem of new urbanization in big urban agglomeration," Human Geography, vol. 4, pp. 1-5, 2015 (Chinese).

[4] C. L. Gu, "Study on urban agglomeration: progress and prospects," Geographical Research, vol. 5, p. 1, 2011 (Chinese).

[5] Y. M. Wang, Y. H. Shi, Y. Fan, X. Y. Jiang, and R. Bai, "Spacetime evolution of the relationship between cities and rivers in the huaihe river upstream region," Journal of Xinyang Normal University, vol. 19, no. 4, pp. 524-528, 2015 (Chinese).

[6] C. Fang, "Important progress and future direction of studies on China's urban agglomerations," Journal of Geographical Sciences, vol. 25, no. 8, pp. 1003-1024, 2015.

[7] B. Mondal, D. N. Das, and G. Dolui, "Modeling spatial variation of explanatory factors of urban expansion of Kolkata: a geographically weighted regression approach," Modeling Earth Systems and Environment, vol. 1, no. 4, pp. 1-13, 2015.

[8] P. Barreira González, F. Aguilera-Benavente, and M. GómezDelgado, "Partial validation of cellular automata based model simulations of urban growth: An approach to assessing factor influence using spatial methods," Environmental Modeling and Software, vol. 69, pp. 77-89, 2015.

[9] A. Rienow and R. Goetzke, "Supporting SLEUTH - Enhancing a cellular automaton with support vector machines for urban growth modeling," Computers, Environment and Urban Systems, vol. 49, pp. 66-81, 2015.

[10] Y. Chen, "Zipf's law, hierarchical structure, and cards-shuffling model for urban development," Discrete Dynamics in Nature and Society, vol. 2012, Article ID 480196, 21 pages, 2012.

[11] Y. Chen, "Urban gravity model based on cross-correlation function and Fourier analyses of spatio-temporal process," Chaos, Solitons \& Fractals, vol. 41, no. 2, pp. 603-614, 2009.

[12] T. Y. Shen, W. D. Wang, M. Hou, Z. Guo, L. Xue, and K. Yang, "Study on Spatio-Temporal system dynamic models of urban growth," Systems Engineering-Theory and Practice, vol. 27, no. 1, pp. 10-17, 2007.

[13] R. B. Thapa and Y. Murayama, "Urban growth modeling of Kathmandu metropolitan region, Nepal," Computers, Environment and Urban Systems, vol. 35, no. 1, pp. 25-34, 2011.

[14] I. E. Ayazli, F. Kilic, S. Lauf, H. Demir, and B. Kleinschmit, "Simulating urban growth driven by transportation networks: A case study of the Istanbul third bridge," Land Use Policy, vol. 49, pp. 332-340, 2015.

[15] Z. Zhang, S. Su, R. Xiao, D. Jiang, and J. Wu, "Identifying determinants of urban growth from a multi-scale perspective: A case study of the urban agglomeration around Hangzhou Bay, China," Applied Geography, vol. 45, pp. 193-202, 2013.

[16] L. M. Bettencourt, J. Lobo, and G. B. West, "Why are large cities faster? Universal scaling and self-similarity in urban organization and dynamics," The European Physical Journal B, vol. 63, no. 3, pp. 285-293, 2008.

[17] S. Hamidi and R. Ewing, "A longitudinal study of changes in urban sprawl between 2000 and 2010 in the United States," Landscape and Urban Planning, vol. 128, pp. 72-82, 2014.

[18] Y. Fan, G. Yu, and Z. He, "Origin, spatial pattern, and evolution of urban system: Testing a hypothesis of "urban tree"," Habitat International, vol. 59, pp. 60-70, 2017.

[19] J. Marull, C. Font, and R. Boix, "Modelling urban networks at mega-regional scale: Are increasingly complex urban systems sustainable?," Land Use Policy, vol. 43, pp. 15-27, 2015. 
[20] E. Abshirini and D. Koch, "Rivers as integration devices in cities," City Territory and Architecture, vol. 3, no. 1, pp. 1-21, 2016.

[21] A. L. J. Ter Wal and R. A. Boschma, "Applying social network analysis in economic geography: Framing some key analytic issues," Annals of Regional Science, vol. 43, no. 3, pp. 739-756, 2009.

[22] Q. Sun, F. Tang, and Y. Tang, "An economic tie networkstructure analysis of urban agglomeration in the middle reaches of Changjiang River based on SNA," Journal of Geographical Sciences, vol. 25, no. 6, pp. 739-755, 2015.

[23] L. C. Freeman, The Development of Social Network Analysis: A Study in the Sociology of Science, Empirical Press, 2004.

[24] F. Nunes, "The Portuguese urban system: An opposition between its hierarchical organization in cyberspace vs. physical space," Telematics and Informatics, vol. 23, no. 2, pp. 74-94, 2006.

[25] C. Reffay and T. Chanier, "How social network analysis can help to measure cohesion in collaborative distance-learning," in Designing for Change in Networked Learning Environments, pp. 343-352, Springer, Dordrecht, Netherlands, 2003.

[26] P. Gloor and. Yan Zhao, "Analyzing Actors and Their Discussion Topics by Semantic Social Network Analysis," in Proceedings of the Tenth International Conference on Information Visualisation (IV'06), pp. 130-135, London, England.

[27] J. Chadchan and R. Shankar, "An analysis of urban growth trends in the post-economic reforms period in India," International Journal of Sustainable Built Environment, vol. 1, no. 1, pp. 36-49, 2012.

[28] G. Grabher, “Trading routes, bypasses, and risky intersections: Mapping the travels of 'networks' between economic sociology and economic geography," Progress in Human Geography, vol. 30, no. 2, pp. 163-189, 2006.

[29] J. Glückler, "Economic geography and the evolution of networks," Journal of Economic Geography, vol. 7, no. 5, pp. 619-634, 2007.

[30] D. Zhu, X. Wu, and J. Zhang, "Application review of gravity model in economic researches," Journal of Yunnan University of Finance and Economics, vol. 5, p. 5, 2008 (Chinese).

[31] Y. Liu, Y. H. Jia, and C. F. Shui, "A method study on layout of intercity transport network: based on gravity model," Human Geography, vol. 6, p. 23, 2011 (Chinese).

[32] X. Li and G. L. Yan, "An empirical research on the characteristics of yangtze river delta urban agglomeration network structure," East China Economic Management, vol. 1, p. 11, 2012 (Chinese).

[33] Y. Hou, Z. Liu, and Z. Yue, "Social Network Analysis over the Process of Economic Integration in the Yangtze River Delta," China Soft Science, vol. 12, pp. 90-101, 2009 (Chinese).

[34] E. Vaz and P. Nijkamp, "Gravitational forces in the spatial impacts of urban sprawl: An investigation of the region of Veneto, Italy," Habitat International, vol. 45, no. 2, pp. 99-105, 2015.

[35] Z. K. Xiao, F. Wang, B. Quan, and L. M. Jiang, "The study of urban interaction based on modified gravity model-A case of Changsha-Zhuzhou-Xiangtan," in Proceedings of the World Automation Congress (WAC '12), pp. 1-4, IEEE, June 2012.

[36] T. Grafeneder-Weissteiner and K. Prettner, "Agglomeration and demographic change," Journal of Urban Economics, vol. 74, no. 1, pp. 1-11, 2013.

[37] N. Kourtellis and A. Iamnitchi, "Leveraging peer centrality in the designof socially-informed peer-to-peer systems," Parallel and Distributed Systems IEEE Transactions, vol. 25, no. 9, pp. 2364-2373, 2012.
[38] J. Liu, Lectures on whole network approach: A practical guide to UCINET, vol. 5-6, Truth and Wisdom Press, Shanghai People's Publishing House, Shanghai, China, 2009.

[39] L. C. Freeman, "Centrality in social networks conceptual clarification," Social Networks, vol. 1, no. 3, pp. 215-239, 1979.

[40] S. P. Borgatti and M. G. Everett, "A graph-theoretic perspective on centrality," Social Networks, vol. 28, no. 4, pp. 466-484, 2006.

[41] W. Luo, P. Yin, Q. Di, F. Hardisty, and A. M. MacEachren, "A geovisual analytic approach to understanding geo-social relationships in the international trade network," PLoS ONE, vol. 9, no. 2, Article ID e88666, 2014.

[42] Y. Wei, H. Liu, W. Song, B. Yu, and C. Xiu, "Normalization of time series DMSP-OLS nighttime light images for urban growth analysis with pseudo invariant features," Landscape and Urban Planning, vol. 128, pp. 1-13, 2014.

[43] D. Li, D. Wang, H. Li, S. Zhang, X. Zhang, and Y. Tao, "The Effects of Urban Sprawl on the Spatial Evolution of Rural Settlements: A Case Study in Changchun, China," Sustainability , vol. 8, no. 8, p. 736, 2016. 


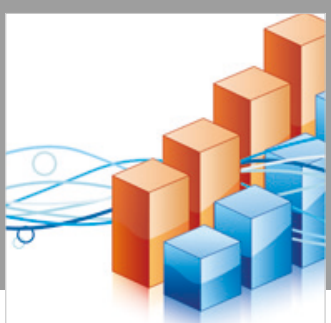

Advances in

Operations Research

\section{-n-m}
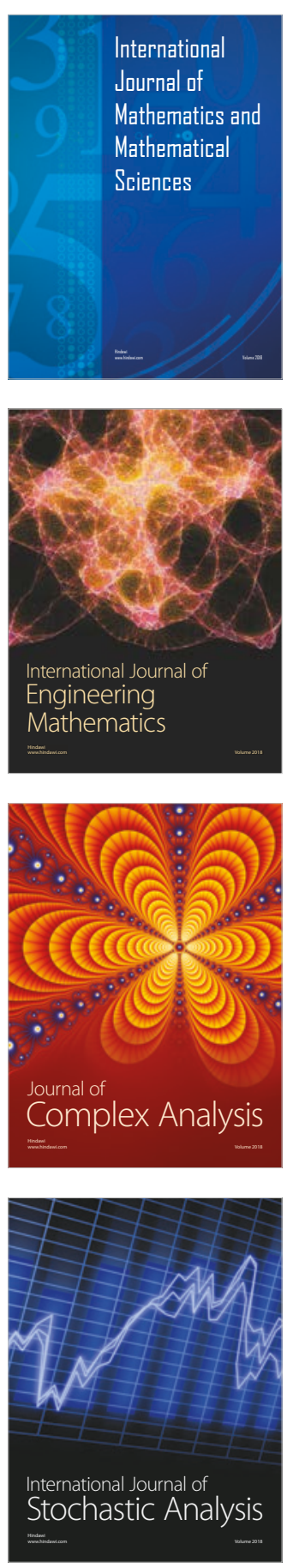
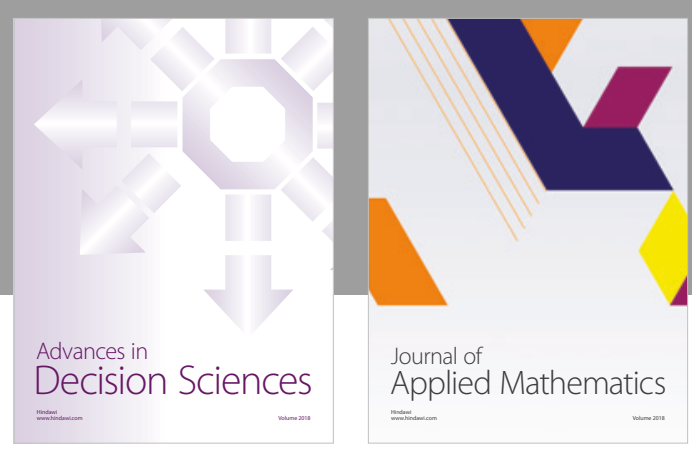

Journal of

Applied Mathematics
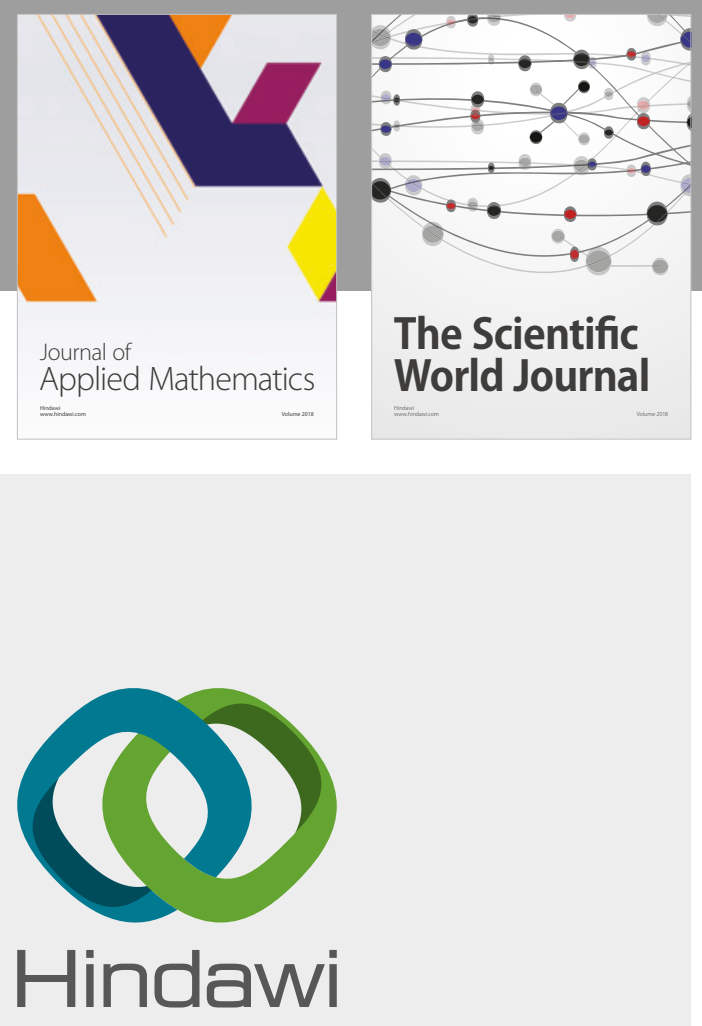

Submit your manuscripts at

www.hindawi.com

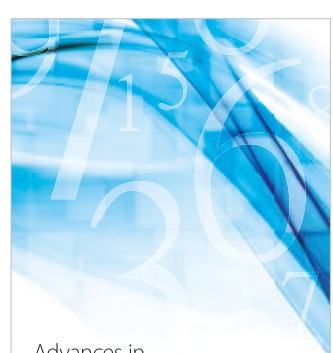

Advances in
Numerical Analysis
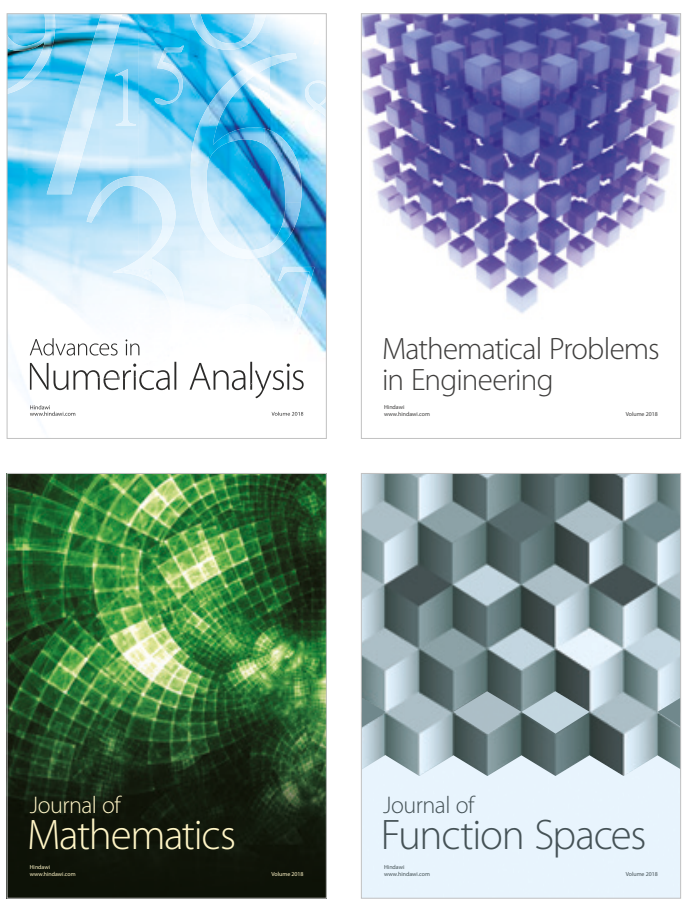

Mathematical Problems in Engineering

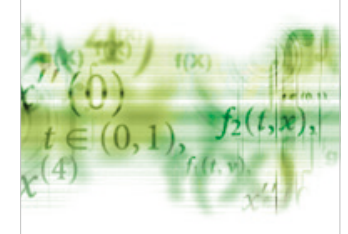

International Journal of

Differential Equations

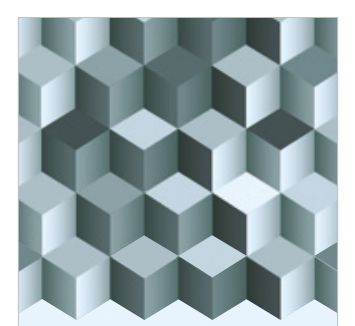

Journal of

Function Spaces

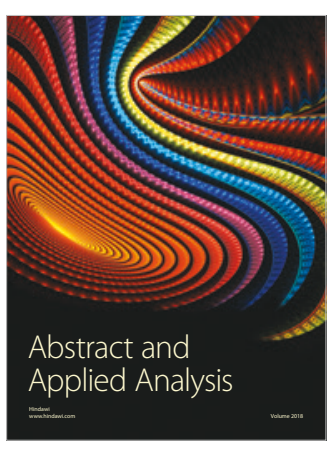

The Scientific

World Journal

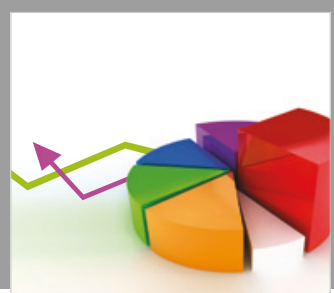

Journal of

Probability and Statistics
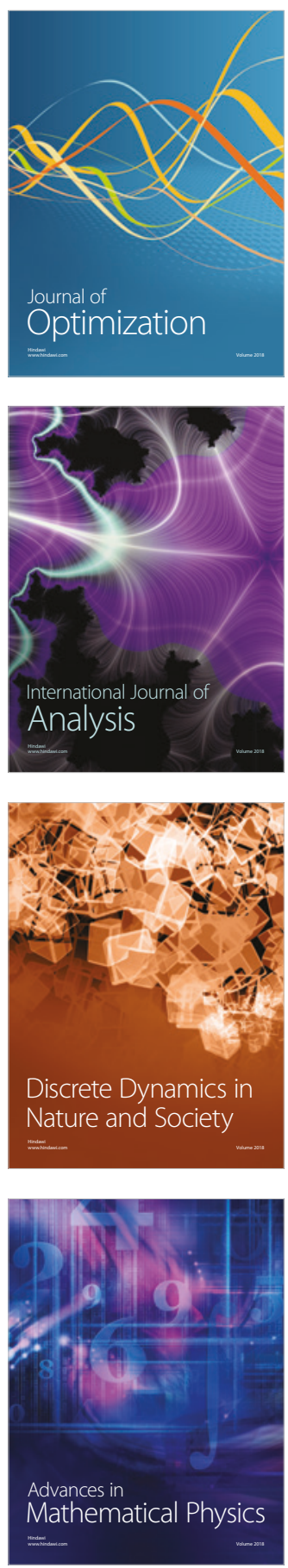